\title{
DETC2007/XXX
}

\section{THE MULTIOBJECTIVE OPTIMIZATION OF A PRISMATIC DRIVE}

\author{
Émilie Bouyer, Stéphane Caro, Damien Chablat \\ Institut de Recherche en Communications \\ et Cybernétique de Nantes \\ UMR CNRS n 6597 \\ 1 rue de la Noë, 44321 \\ Nantes, France \\ Email: \{stephane.caro, damien.chablat\}@irccyn.ec-nantes.fr
}

\author{
Jorge Angeles \\ Department of Mechanical Engineering \& \\ Centre for Intelligent Machines \\ McGill University \\ 817 Sherbrooke West, H3A 2K6, \\ Montreal, QC, Canada \\ Email: angeles@cim.mcgill.ca
}

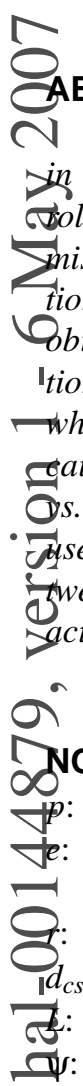

\section{ABSTRACT}

The multiobjective optimization of Slide-o-Cam is reported in this paper. Slide-o-Cam is a cam mechanism with multiple bollers mounted on a common translating follower. This transmission provides pure-rolling motion, thereby reducing the friction of rack-and-pinions and linear drives. A Pareto frontier is Obtained by means of multiobjective optimization. This optimiza'tion is based on three objective functions: (i) the pressure angle, which is a suitable performance index for the transmission because it determines the amount of force transmitted to the load s. that transmitted to the machine frame; (ii) the Hertz pressure Hsed to evaluate the stresses produced on the contact surface beween cam and roller; and (iii) the size of the mechanism, characterized by the number of cams and their width.

\section{NOMENCLATURE}

pitch of the transmission;

distance between the axis of the cam and the line of centers of the rollers;

radius of the roller;

diameter of the camshaft $\left(d_{c s}=2(e-r)\right)$;

the width of the contact between the cams and the rollers;

input of the mechanism, i.e., the angle of rotation of the cam;

$s:$ output of the mechanism, i.e., the displacement of the follower;

$\mu$ : pressure angle; f: force transmitted from the cam to the roller;

$\kappa_{c}$ and $\kappa_{p}$ : curvature of the cam profile and the pitch curve, respectively;

$\rho_{c}$ and $\rho_{p}$ : radii of curvature of the cam profile and the pitch curve, respectively;

$m$ : number of cams mounted on the camshaft;

$n$ : number of lobes per cam;

$P$ : Hertz pressure;

$S_{M}:$ size of the mechanism.

\section{INTRODUCTION}

In robotic and mechatronic applications, whereby motion is controlled using a piece of software, the conversion from rotational to translational motion is usually realized by means of ball-screws or linear actuators. While both are gaining popularity, they present some drawbacks. On the one hand, ball-screws comprise a high number of moving parts, their performance depending on the number of balls rolling in the shaft groove. Moreover, they have a low load-carrying capacity due to the punctual contact between balls and groove. On the other hand, linear bearings are composed of roller-bearings to figure out the previous issue, but these devices rely on a form of direct-drive motor, which makes them expensive to produce and maintain.

A novel transmission, called Slide-o-Cam, is depicted in Fig. 1] as introduced in [1] to transform a rotational motion into a translational one. Slide-o-Cam is composed of four main elements: (i) the frame; (ii) the cam; (iii) the follower; and (iv) the 
rollers. The input axis on which the cams are mounted, named camshaft, is driven at a constant angular velocity by means of an actuator under computer-control. Power is transmitted to the output, the translating follower, which is the roller-carrying slider, by means of pure-rolling contact between the cams and the rollers. The roller comprises two components, the pin and the bearing. The bearing is mounted to one end of the pin, while the other end is press-fit into the roller-carrying slider. Consequently, the contact between the cams and rollers occurs at the outer surface of the bearing. The mechanism uses two conjugate cam-follower pairs, which alternately take over the motion transmission to ensure a positive action; the rollers are thus driven by the cams throughout a complete cycle. Therefore, the main advantages of cam-follower mechanisms with respect to the other transmissions, which transform rotation into translation are: (i) lower friction; (ii) higher stiffness; (iii) low backlash; and (iv) reduction of wear. The multiobjective optimization of

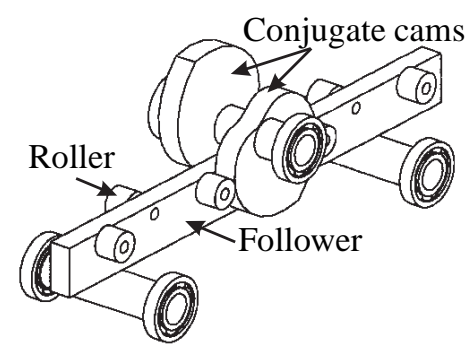

Figure 1. Layout of Slide-o-Cam

Slide-o-Cam is reported in this paper. This optimization is based on three criteria: $(i)$ the pressure angle, a suitable performance index for the transmission because it determines the amount of force transmitted to the load vs. that transmitted to the machine frame; (ii) the Hertz pressure, a measure of the stresses produced in the contact surface between the cams and the rollers; and (iii) the size of the mechanism, characterized by the number of cams and their width.

\section{SYNTHESIS OF PLANAR CAM MECHANISMS}

Let the $x-y$ frame be fixed to the machine frame and the $u-v$ frame be attached to the cam, as depicted in Fig. 2. $O_{1}$ is the origin of both frames, $\mathrm{O}_{2}$ is the center of the roller, and $\mathrm{C}$ is the contact point between cam and roller. The geometric parameters are illustrated in the same figure. The notation used in this figure is based on the general notation introduced in [2币, namely, $(i)$ the pitch $p$, i.e., the distance between the center of two rollers on the same side of the follower; (ii) the distance $e$ between the axis of the cam and the line of centers of the rollers; (iii) the radius $r$ of

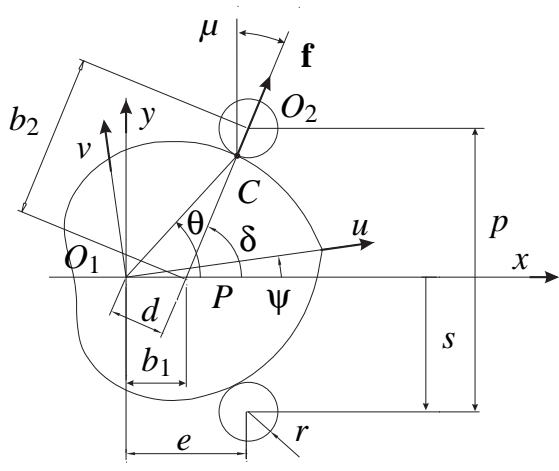

Figure 2. Parameterization of Slide-o-Cam

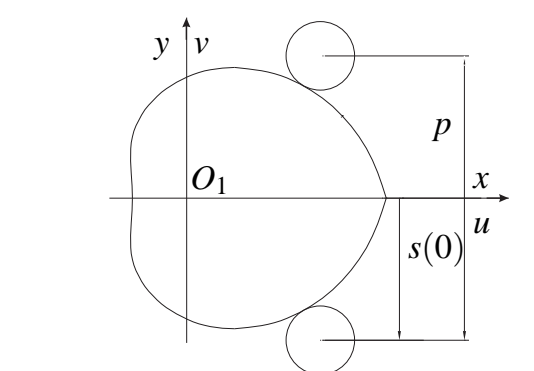

Figure 3. Home configuration of the mechanism

the roller-bearing, i.e., the radius of the roller; (iv) the angle of rotation $\psi$ of the cam, the input of the mechanism; $(v)$ the position $s$ of the center of the roller, i.e, the displacement of the follower, which is the output of the mechanism; (vi) the pressure angle $\mu$; and (vii) the force $\mathbf{f}$ transmitted from the cam to the roller.

The above parameters as well as the surface of contact on the cam are determined by the geometric relations derived from the Aronhold-Kennedy Theorem [2]]. As a matter of fact, when the cam makes a complete turn, i.e., $\Delta \psi=2 \pi$, the displacement of the roller is equal to the pitch, i.e., $\Delta s=p$. Furthermore, if we consider that Fig. 3 illustrates the home configuration of the roller, the latter is below the $x$-axis when $\psi=0$. Therefore, $s(0)=-p / 2$ and the input-output function $s$ is defined as:

$$
s(\psi)=\frac{p}{2 \pi} \psi-\frac{p}{2}
$$

The cam profile is determined by the displacement of the contact point $C$ around the cam. The Cartesian coordinates of $C$ in the $u-v$ frame take the form [5]

$$
u_{c}(\psi)=b_{1} \cos \psi+\left(b_{2}-r\right) \cos (\delta-\psi)
$$




$$
v_{c}(\psi)=-b_{1} \sin \psi+\left(b_{2}-r\right) \sin (\delta-\psi)
$$

The expressions of coefficients $b_{2}, b_{3}$ and $\delta$, as obtained in 6 10], are:

$$
\begin{aligned}
b_{1} & =\frac{p}{2 \pi} \\
b_{2} & =\frac{p}{2 \pi} \sqrt{(2 \pi \eta-1)^{2}+(\psi-\pi)^{2}} \\
\delta & =\arctan \left(\frac{\psi-\pi}{2 \pi \eta-1}\right)
\end{aligned}
$$

where $\eta=e / p$, a nondimensional design parameter.

From Eq.(3c), we can notice that $\eta$ cannot be equal to $1 /(2 \pi)$. Moreover, an extended angle $\Delta$ was introduced in [6]

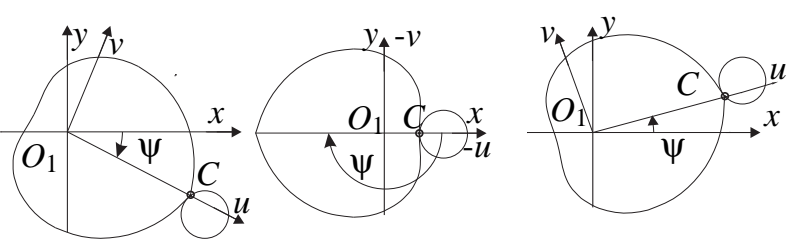

(a)

(b)

(c)

Figure 4. Orientations of the cam found when $v_{c}=0$ : (a) $\psi=\Delta$; (b) $\psi=\pi ;$ and (c) $\psi=2 \pi-\Delta$

to obtain a closed cam profile. $\Delta$ is defined as a root of Eq. (2b). As far as Slide-o-Cam is concerned, $\Delta$ is negative, as shown in Fig. 4 . Consequently, to close the cam profile, $\psi$ must vary within $\Delta \leq \psi \leq 2 \pi-\Delta$.

\subsection{Pitch-Curve Determination}

The pitch curve is the trajectory of $\mathrm{O}_{2}$, the center of the roller, distinct from the trajectory of the contact point $C$, which produces the cam profile. The Cartesian coordinates $(e, s)$ of point $\mathrm{O}_{2}$ in the $x-y$ frame are depicted in Fig. 2. Hence, the Cartesian coordinates of the pitch-curve in the $u-v$ frame are

$$
\begin{aligned}
& u_{p}(\psi)=e \cos \psi+s(\psi) \sin \psi \\
& v_{p}(\psi)=-e \sin \psi+s(\psi) \cos \psi
\end{aligned}
$$

\subsection{Curvature of the Cam Profile}

The curvature $\kappa_{p}$ of the pitch curve is given in [10] as

$$
\kappa_{p}=\frac{2 \pi}{p} \frac{\left[(\psi-\pi)^{2}+2(2 \pi \eta-1)(\pi \eta-1)\right]}{\left[(\psi-\pi)^{2}+(2 \pi \eta-1)^{2}\right]^{3 / 2}}
$$

provided that the denominator does not vanish at any value of $\psi$ within $\Delta \leq \psi \leq 2 \pi-\Delta$, i.e., $\eta \neq 1 /(2 \pi)$.

Let $\rho_{c}$ and $\rho_{p}$ be the radii of curvature of the cam profile and the pitch curve, respectively, and $\kappa_{c}$ the curvature of the cam profile. Since the curvature is the reciprocal of the radius of curvature, we have $\rho_{c}=1 / \kappa_{c}$ and $\rho_{p}=1 / \kappa_{p}$. Furthermore, due to the definition of the pitch curve, it is apparent that

$$
\rho_{p}=\rho_{c}+r
$$

From Eq. (6), the curvature of the cam profile can be written as

$$
\kappa_{c}=\frac{\kappa_{p}}{1-r \kappa_{p}}
$$

In [9], the authors claimed that the cam profile has to be fully convex for machining accuracy. Such a profile can be obtained if and only if $\eta>1 / \pi$. In order to increase the range of design parameters, we include non-convex cams within the scope of this paper. Nevertheless, the sign of the local radius $\rho_{c}$ has to remain positive as long as the cam pushes the roller. In this vein, the cam is convex when $\eta \in] 1 /(2 \pi), 1 / \pi]$ and $\psi \in] \Delta, \pi][11]$. Moreover,
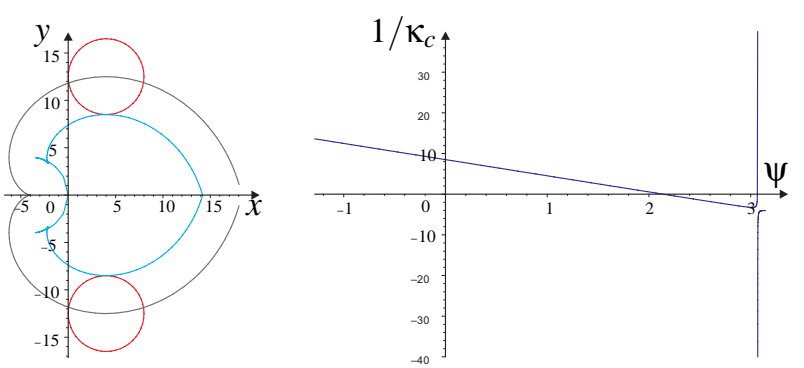

Figure 5. Cam profile and local curvature of the cam

according to [9], $\rho_{c}$ is a minimum when

$$
\psi=\psi_{\min }=\frac{\pi-\sqrt{4 n^{2} \pi h-n^{2}-4 n^{2} \pi^{2} h^{2}}}{n}
$$

where $n$ is the number of lobes per cam. Therefore, the cam profile is not feasible when $\rho_{c}\left(\psi_{\min }\right)<0$. If this inequality becomes an equality, the roller will block the cam, as depicted in Fig. 5.

\section{MULTIOBJECTIVE OPTIMIZATION PROBLEM}

We introduce in this section the multiobjective optimization of Slide-o-Cam. Indeed, such an optimization is needed to prop- 
erly dimension the mechanism. First, the objective functions are defined. Then, a sensitivity analysis of the mechanism is reported in order to choose shrewdly the design variables of the optimization problem. Finally, the results of the latter are illustrated by means of a Pareto frontier as the objective functions are antagonistic.

\subsection{The Objective Functions}

The optimization of the mechanism is based on three objective functions: $(i)$ the maximum pressure angle $\mu_{\max }$; $(i i)$ the maximum Hertz pressure $P_{\max }$ related to the contact between the cams and the rollers; and ( $\mathrm{iii}$ ) the size of the mechanism $S_{M}$. As a matter of fact, we want to simultaneously minimize these three functions.

3.1.1 The Pressure Angle The pressure angle $\mu$ of a cam-roller-follower mechanism is defined as the angle between the normal to the contact point $C$ between the cam and the roller and the velocity of $C$ as a point of the follower [3]. As illustrated in Fig. 2, $\mu$ is a significant parameter in cam design. In fact, the smaller $\mu^{1}$, the better the transmission. The expression for $\mu$ is given in [3]; in terms of $\eta$, we have

$$
\tan \mu=\frac{n-2 n \pi \eta}{n \psi-\pi}
$$

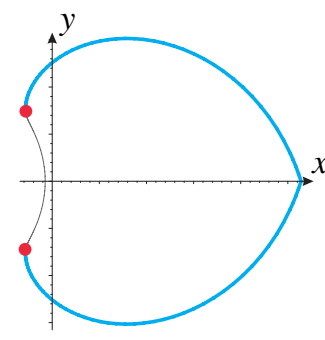

(a)

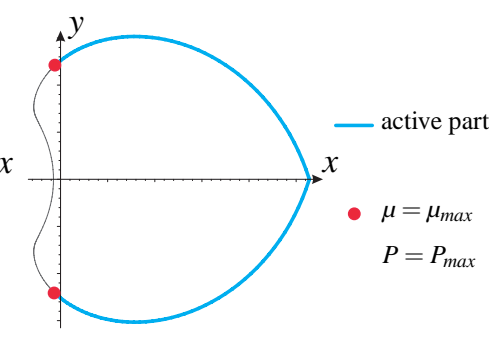

(b)
Figure 6. Active parts of: (a) a two- and (b) three-conjugate cam mechanisms

Figure 6 illustrates the active parts of a two- and a threeconjugate-cam mechanisms. It turns out that the pressure angle is a maximum at the ends of the active parts for the two mechanisms. In this paper, $\mu_{\max }$ denotes the maximum pressure angle

\footnotetext{
${ }^{1} \mu$ is a real number and can be either positive or negative. However, within the scope of this paper, $\mu$ remains positive. Therefore, $\mu=|\mu|,|\cdot|$ denoting the absolute value.
}

along the active part of the cam profile; it is an objective function in this optimization problem.

3.1.2 The Hertz Pressure When two bodies with curved surfaces, for example, a cam and a roller, are pressed together, contact takes place not along a line but along a surface, due to the inherent material compliance. Moreover, the stresses developed in the two bodies are three-dimensional. Those contact stresses may generate failures as cracks, pits, or flaking in the surface material. To quantify these stresses, Heinrich

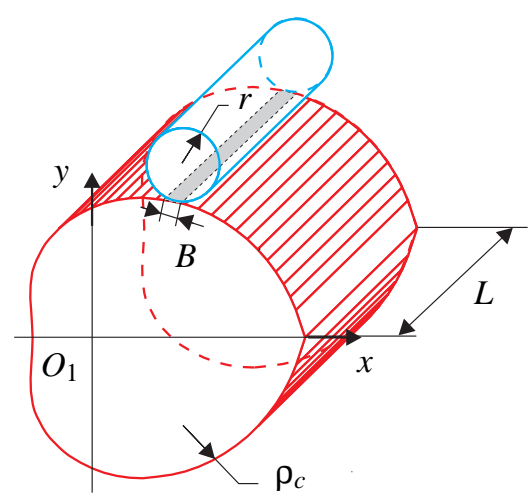

Figure 7. The width $B$ of the contact between a cam and a roller

Rudolf Hertz (1857-1894) proposed some formulas to evaluate the width of the band of contact between two cylinders and the maximum pressure of contact, called Hertz pressure. In Slide-oCam, the rollers and the cams are the bodies in contact. Unlike the roller, the cam is not a cylinder, but can be approximated by a cylinder with radius identical to the radius of curvature of the cam at the contact point. The width $B$ of the band of contact is illustrated in Fig.7, and given by Hertz as

$$
\begin{aligned}
B & =\sqrt{\frac{16 F\left(K_{1}+K_{2}\right) R_{\text {equ }}}{L}} \\
R_{\text {equ }} & =\frac{r \rho_{c}}{r+\rho_{c}}
\end{aligned}
$$

$F$ being the magnitude of the axial load $\mathbf{f}$ while $R_{\text {equ }}$ is the equivalent radius of contact, $L$ the width of the contact between the cams and the rollers, and $K_{1}$ and $K_{2}$ the coefficients that characterize the materials of the cams and the rollers, respectively, i.e.,

$$
K_{1}=\frac{1-v_{1}^{2}}{\pi E_{1}}, \quad K_{2}=\frac{1-v_{2}^{2}}{\pi E_{2}}
$$


where $v_{1}$ and $v_{2}$ are the Poisson ratios of the materials of the cam and the roller, respectively, while $E_{1}, E_{2}$ their corresponding Young moduli. Accordingly, the Hertz pressure $P$ of the contact between the cams and the rollers takes the form:

$$
P=\frac{4 F}{L \pi B}
$$

Let us notice that $P$ depends on $\psi$, as $F$ is a function of this variable and $B$ is a function of $F$.

Let us assume that $F$ is constant. As $L$ and $r$ are constant and $R_{\text {equ }}$ is monotonic with respect to (w.r.t) $\rho_{c}$ as long as $\rho_{c}>-r$, from Eq. 10a), the lower $\rho_{c}$, the lower $B$. From Eq.(12), the lower $B$, the higher $P$. According to [11], $\rho_{c}$ is a minimum when $\psi=\pi / n-\Delta$ for a two-conjugate cam mechanism. Therefore, $P$ is a maximum when $\psi=\pi / n-\Delta$ for such a mechanism.

Figure 6 illustrates the active parts of a two- and a threeconjugate-cam mechanisms. It turns out that the Hertz pressure is a maximum at the ends of the active parts for the two mechanisms as $\rho_{c}$ is a minimum at those ends. In this paper, $P_{\max }$ denotes the maximum Hertz pressure along the active part of the cam profile; it is an objective function in this optimization problem.

The maximum Hertz pressures allowed for some materials are obtained from [12] and recorded in Table 1. The second column gives the allowable pressure $P_{\text {stat }}$ for a static load. As a matter of fact, it is recommended not to apply more than $40 \%$ of $P_{\text {stat }}$ in order to secure an infinite fatigue life. The corresponding values $P_{\text {max }}$ are given in the third column of Table 1. Obviously,

Table 1. Allowable pressures

\begin{tabular}{ccc}
\hline \hline Material & $P_{\text {stat }}[\mathrm{MPa}]$ & $P_{\max }[\mathrm{MPa}]$ \\
\hline Stainless steel & 650 & 260 \\
Improved steel & 1600 to 2000 & 640 to 800 \\
Grey cast iron & 400 to 700 & 160 to 280 \\
Aluminum & 62.5 & 25 to 150 \\
Polyamide & 25 & 10 \\
\hline \hline
\end{tabular}

the maximum allowable pressure depends also on the shape of the different parts in contact. A thick part will be stiffer than a thin one. Nevertheless, we only take into account the material of the cams and rollers for the determination of the allowable pressures within the scope of this research work. Finally, let us notice that only improved steel is appropriate for a Slide-o-Cam transmission in case of high Hertz-pressure values.
3.1.3 Size The size of the mechanisms $S_{M}$ is defined as

$$
S_{M}=m L
$$

where $m$ is the number of cams. From [9], a Slide-o-Cam with only one cam, i.e., $m=1$, is not feasible. Besides, the smaller $S_{M}$, the less bulky the mechanism.

\subsection{The Design Variables}

The design variables of the optimization problem are: $(i)$ the diameter $d_{c s}$ of the camshaft $\left(d_{c s}=e-r\right)$; (ii) the radius $r$ of the rollers; (iii) the width $L$ of the contact between cam and roller; and (iv) the number of cams $m$.

\subsection{Sensitivity Analysis}

We conduct here the analysis of the sensitivity of the performance of Slide-o-Cam to the variations in its design parameters. Such an analysis is needed to both determine the tolerance of the design variables and obtain a robust design.

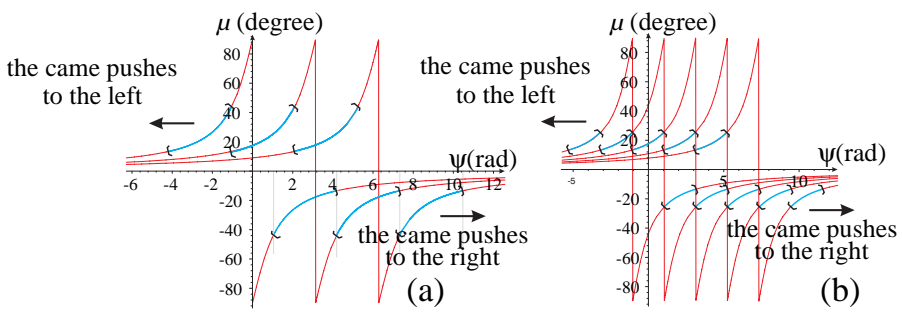

Figure 8. Pressure-angle distribution for (a) two conjugate-cam and (b) three conjugate-cam mechanisms with one lobe, $p=50, r=10$ and $e=9$

3.3.1 Sensitivity of the Pressure Angle Figure 8 illustrates the pressure-angle distribution for two conjugate- and three conjugate-cams with one lobe. We can notice that the pressure angle decreases with the number of cams. Consequently, we can use conjugate cams, namely, several cams mounted on the camshaft, to reduce the pressure angle. Below is a list of the effects of some design parameters on the pressure angle:

1. The lower $\eta$, the lower the pressure angle, with $\eta \geq 1 / \pi$;

2. the lower $r$, the lower the pressure angle;

3 . the lower $n$, the lower the pressure angle, [9];

4. the higher $m$, the lower the pressure angle.

where $m$ is the number of cam(s) mounted on the camshaft.

As the pressure angle increases with the number of lobes, we consider only mono-lobe cams, i.e. $n=1$. 
3.3.2 Sensitivity of the Hertz Pressure $P_{\max }$ depends on the geometry of the cam, the number of conjugate cams, the material of the parts in contact and the load applied. Therefore, we have different ways to minimize the Hertz pressure, namely,

1. The higher $m$, the lower $P_{\max }$;

2. the lower the axial load, the lower $P_{\max }$;

3 . the more compliant the material, the lower $P_{\max }$;

4. the higher $L$, the lower $P_{\max }$.

In order to analyze the sensitivity of $P$ to $r, \eta, p$ and $L$, we use a first derivative model of $P$ w.r.t. the corresponding parameters, i.e.,

$$
\delta P \approx \mathbf{c} \delta \mathbf{q}
$$

with

$$
\mathbf{c}=\left[\begin{array}{l}
\partial P / \partial r \\
\partial P / \partial \eta \\
\partial P / \partial p \\
\partial P / \partial L
\end{array}\right], \delta \mathbf{q}=\left[\begin{array}{l}
\delta r \\
\delta \eta \\
\delta p \\
\delta L
\end{array}\right]
$$

If the values of the parameters are known, we will be able to evaluate c. Let us assume that $r=4 \mathrm{~mm}, \eta=0.18, p=50 \mathrm{~mm}$ and $L=10 \mathrm{~mm}$. The partial derivatives have to be normalized to

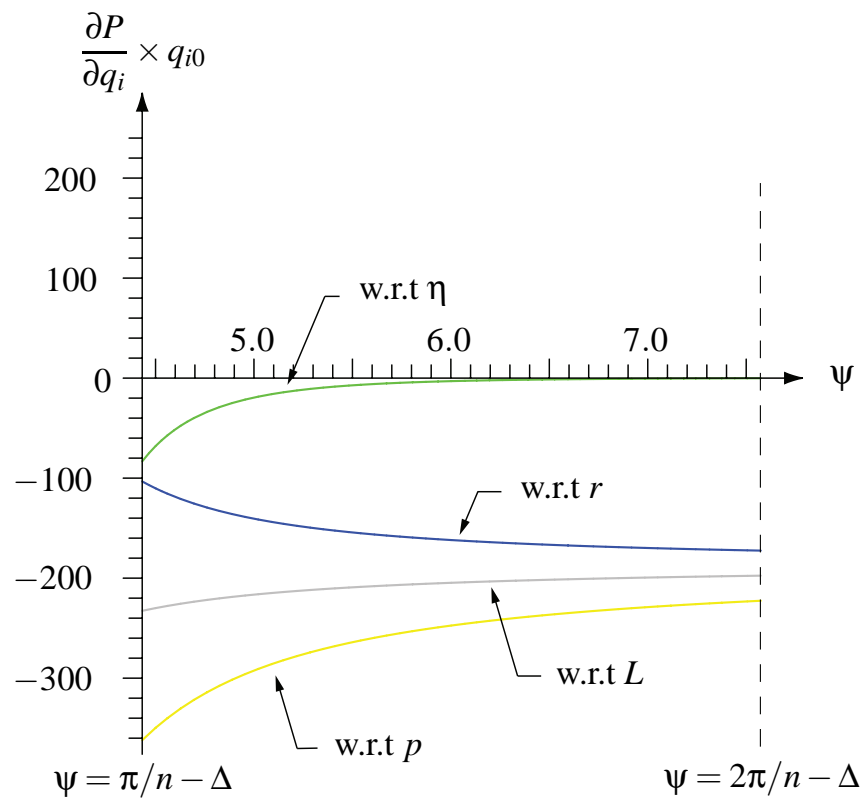

Figure 9. Influence of the variations in $r, \eta, p$ and $L$ on $P$ be compared. In this vein, we divide each of them by its nominal value. Now, we can plot each partial derivative with respect to the angle of rotation of the cam $\psi$, as illustrated in Fig. 9

The most influential variables are those with the highest absolute value of their corresponding partial derivative for a given value of $\psi$. As the maximum value of the Hertz pressure is obtained for $\psi=\pi / n-\Delta$ and $\Delta=-1.2943 \mathrm{rad}$, the partial derivatives can be evaluated for $\psi=\pi / n-\Delta$. The sensitivity of $P_{\max }$ to $\delta \mathbf{q}$ is recorded in Table 2. The plots in Fig. 8 show the sensitivity

Table 2. Influence of the variations in $r, \eta, p$ and $L$ on $P_{\max }$

\begin{tabular}{ccccc}
\hline \hline$q_{i}$ & $r$ & $\eta$ & $p$ & $L$ \\
\hline$q_{\text {init }}$ & $4 \mathrm{~mm}$ & 0.18 & $50 \mathrm{~mm}$ & $10 \mathrm{~mm}$ \\
$\left|\frac{\partial P_{\text {max }}}{\partial q_{i}}\left(q_{\text {init }}\right)\right|$ & 103.32 & 83.25 & 362.03 & 232.67 \\
Order of importance & 3 & 4 & 1 & 2 \\
\hline \hline
\end{tabular}

of the Hertz pressure w.r.t the different parameters for different values of $\psi$ and for the active part of the cam profile. However, it is more relevant to calculate the rms value of each partial derivative, as recorded in Table 3. As a matter of fact, Table 2 and

Table 3. Global influence of the variations in $r, \eta, p$ and $L$ on $P$

\begin{tabular}{ccccc}
\hline \hline$q_{i}$ & $r$ & $\eta$ & $p$ & $L$ \\
\hline$q_{\text {init }}$ & 4 & 0.18 & 50 & 10 \\
$\sqrt{\frac{n}{\pi} \int_{\psi}\left(\frac{\partial P}{\partial q_{i}}\right)^{2} \mathrm{~d} \psi}$ & 156.59 & 20.21 & 261.85 & 207.79 \\
Order of importance & 3 & 4 & 1 & 2 \\
\hline \hline
\end{tabular}

Table 3 provide the same results in terms of order of importance of the variations in $r, \eta, p$ and $L$. Finally, in order to minimize the variations in the Hertz pressure, we had better minimize the variations in $p, L r$ and $\eta$ in descending order.

\subsubsection{Sensitivity of the Size of the Mechanism} The sensitivity analysis of $S_{M}$ is trivial. Indeed, from eq. (13), the higher $m$, the higher $S_{M}$. Likewise, the higher $L$, the higher $S_{M}$.

\subsection{Problem Formulation}

A motivation of this research work is to implement a Slideo-Cam transmission in the Orthoglide, a low-power machine tool 
introduced in [8]. To that end, the transmission has to transmit a torque $C_{t}$ of $1.2 \mathrm{Nm}$ with a pitch of $20 \mathrm{~mm}$. In case of high-speed operations, i.e., when the velocity of the cams is higher than $50 \mathrm{rpm}$, the pressure-angle is recommended to be smaller than $30^{\circ}$. Table 1 shows that the maximum value of the Hertz pressure has to be smaller than $800 \mathrm{MPa}$ as the cams and the rollers are made up of steel. Moreover $S_{M}$ is supposed to be smaller than $90 \mathrm{~mm}$ with a view to limiting the size of the mechanism. Besides, the Slide-o-Cam transmissions under study are composed of two- or three- conjugate cams as a Slide-o-Cam with only one cam is not feasible and such a mechanism with more than three conjugate cams would be too bulky, i.e., $m=\{2,3\}$. Consequently, the optimization problem can be formulated

$$
\begin{aligned}
& \qquad \min _{\mathbf{x}}\left(\mu_{\max }, P_{\max }, S_{M}\right) \\
& \text { s.t. } \\
& \mu_{\max } \leq 30^{\circ} \\
& P_{\max } \leq 800 \mathrm{MPa} \\
& S_{M} \leq 90 \mathrm{~mm} \\
& \mathbf{x}_{l} \leq \mathbf{x} \leq \mathbf{x}_{u}
\end{aligned}
$$

where $\mathbf{x}=\left[d_{c s}, r, L, m\right]^{T}$, while $\mathbf{x}_{l}$ and $\mathbf{x}_{u}$ denote the lower and upper bounds of the design variables, respectively. Here, $\mathbf{x}_{l}=$ [0 mm, $4 \mathrm{~mm}, 0 \mathrm{~mm}, 2]$ and $\mathbf{x}_{u}=\left[0 \mathrm{~mm}, 10.5 \mathrm{~mm}, L_{\max }, 3\right]$, $L_{\max }$ being equal to $S_{M a x} / m$ knowing that $S_{M a x}=90 \mathrm{~mm}$.

\subsection{Results}

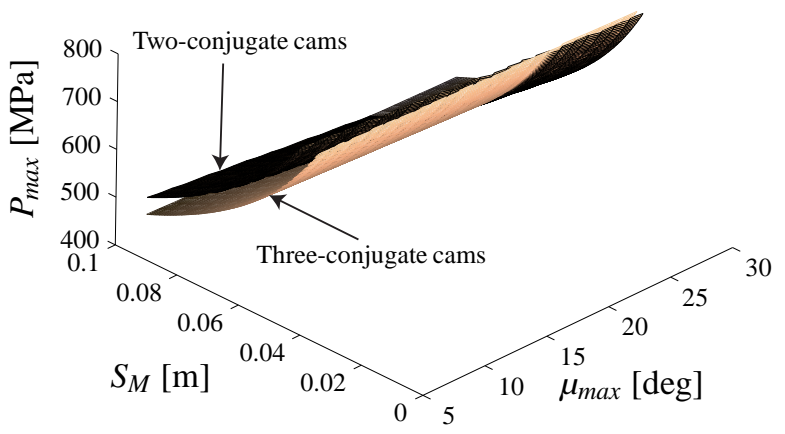

Figure 10. Pareto frontier of a two- and a three- conjugate cam mechanisms

The optimization problem defined in Section 3.4 is multiobjective with objective functions of a different nature. For this reason, the optimum solutions of the problem can be illustrated

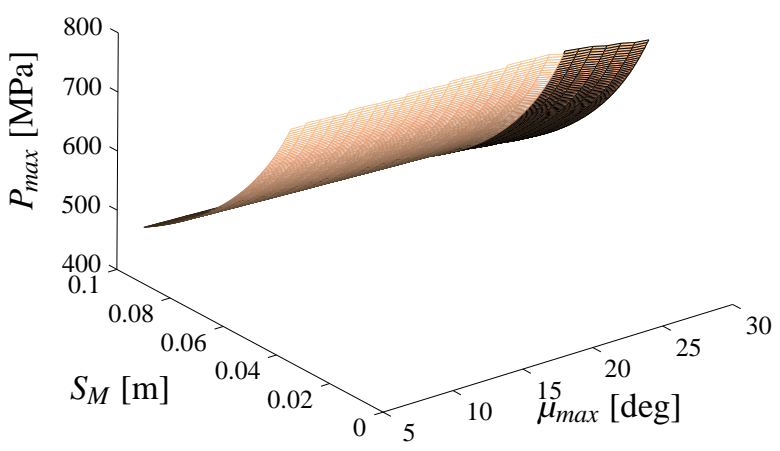

Figure 11. Pareto frontier of Slide-o-Cam mechanisms

by means of a Pareto frontier [13]. As the problem involves three objective functions, i.e., $\mu_{\max }, P_{\max }$ and $S_{M}$, the corresponding Pareto frontier is depicted in 3D space as shown in Figs. 10 and 11. Figure 10 illustrates the Pareto frontiers of a two- and a threeconjugate cam mechanisms. As we want to minimize the three objective functions concurrently, the closer the Pareto frontier to the origin, the better the design. In Fig. 10, we notice that the optimum solutions obtained with a three-conjugate cam mechanism are slightly better when $\mu_{\max }$ is smaller than $24^{\circ}$. Otherwise, a two-conjugate cam mechanism turns out to be more interesting. Nevertheless, the difference between the optimum solutions obtained with a two- and a three-conjugate cam mechanisms remains low. Figure 11 depicts the region closest to the origin of the two frontiers shown in Fig. 10. It also shows the Pareto frontier of Slide-o-Cam mechanisms, regardless of the number of conjugate-cams.

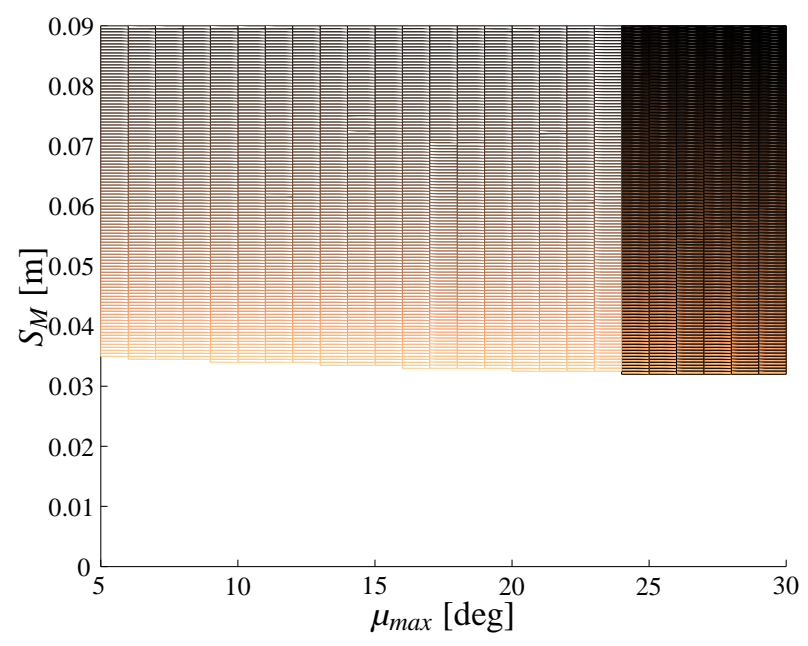

Figure 12. Pareto frontier w.r.t. $\mu_{\max }$ and $S_{M}$ 


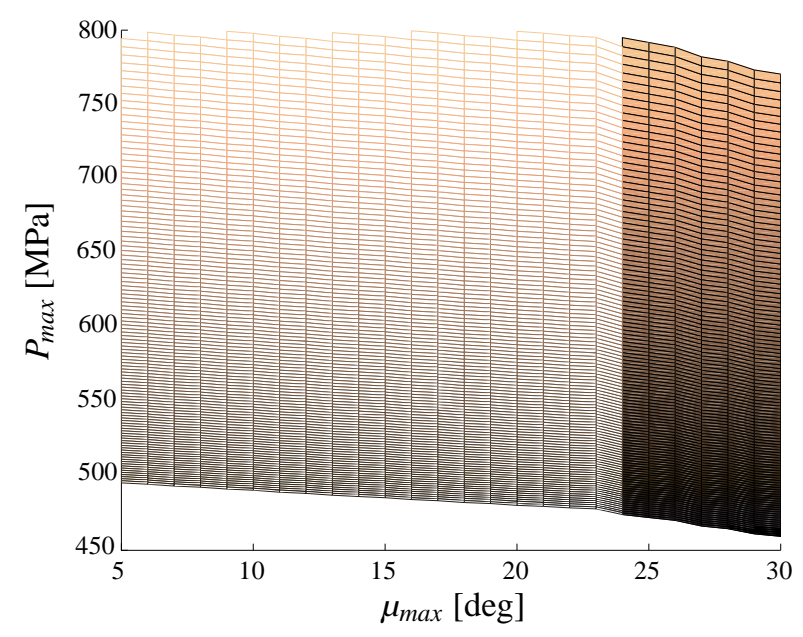

Figure 13. Pareto frontier w.r.t. $P_{\max }$ and $\mu_{\max }$

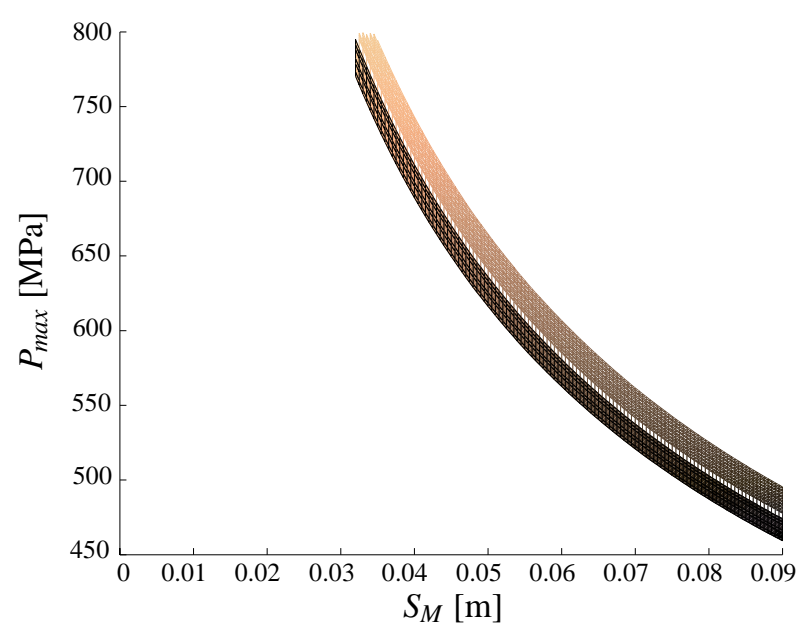

Figure 14. Pareto frontier w.r.t. $P_{\max }$ and $S_{M}$

For better clarity of the results, Figs. 12, 13 and 14 illustrate the projections of the Pareto frontier shown in Fig. 11 w.r.t $\mu_{\max }$ and $S_{M} ; P_{\max }$ and $\mu_{\max }$; and $P_{\max }$ and $S_{M}$, respectively. These figures allow us to see clearly the location the optimum and the feasible solutions of the problem at hand.

Figures 15 and 16 illustrate the contours of $\mu_{\max }$ and $P_{\max }$ w.r.t $d_{c S}$ and $r$ for a two- and a three-conjugate cam mechanisms with $S_{M}=0.06 \mathrm{~m}$. On the one hand, the continuous lines depict the iso-contours of $\mu_{\max }$. On the other hand, the broken lines depict the iso-contours of $P_{\max }$. Besides, Figs. 15 and 16 highlight the location of the optimum solutions for a two- and a threeconjugate cam mechanisms with $S_{M}=0.06 \mathrm{~m}$. We can notice

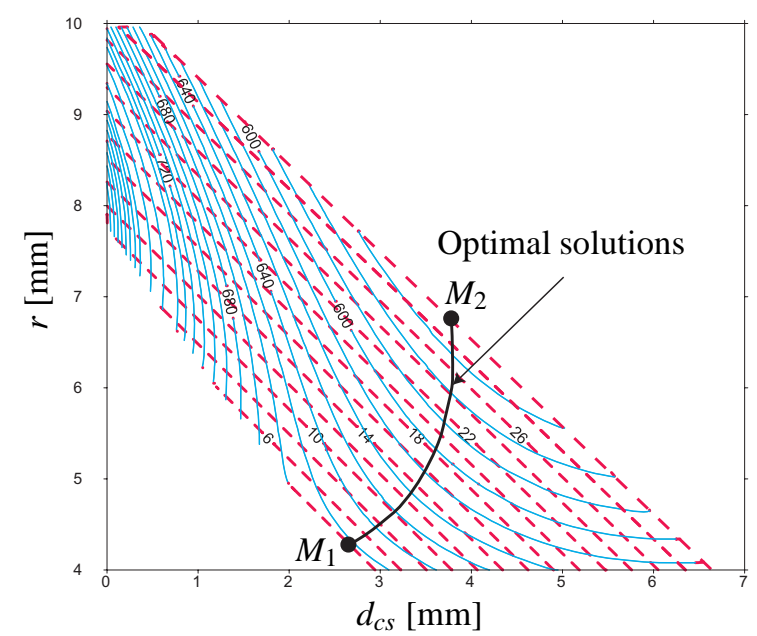

Figure 15. Contours of $\mu$ and $P$ w.r.t $d_{c s}$ and $r$ and the location of the optimal solutions for a two conjugate-cam mechanism with $S_{M}=0.06 \mathrm{~m}$

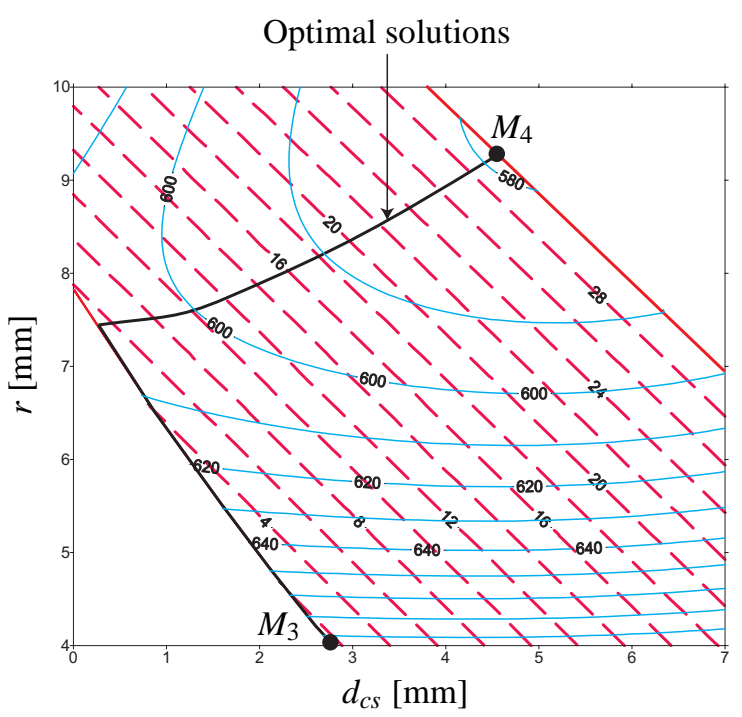

Figure 16. Contours of $\mu$ and $P$ w.r.t. $d_{c s}$ and $r$ and the location of the optimal solutions for a three conjugate-cam mechanism with $S_{M}=$ $0.06 \mathrm{~m}$

that the line of optimum solutions in the space of design variables $d_{c s}$ and $r$ is longer in Fig. 16 than in Fig. 15. This means that a three-conjugate cam mechanism allows more optimal solutions than its two-conjugate cam counterpart. In this vein, it is more interesting to design a three-conjugate cam mechanism.

Figure 17 depicts the mechanisms corresponding to points $M_{1}$ and $M_{2}$ that are plotted in Fig.15. For $M_{1}, d_{c s}=2.6 \mathrm{~mm}$, $r=4.24 \mathrm{~mm}, \mu_{\max }=3^{\circ}$ and $P_{\max }=653.83 \mathrm{MPa}$. For $M_{2}, d_{c s}=$ $4.16 \mathrm{~mm}, r=6.4 \mathrm{~mm}, \mu_{\max }=30^{\circ}$ and $P_{\max }=562.12 \mathrm{MPa}$.

Figure 18 depicts the mechanisms corresponding to points $M_{3}$ and $M_{4}$ that are plotted in Fig.16. For $M_{3}, d_{c s}=2.2 \mathrm{~mm}$, 

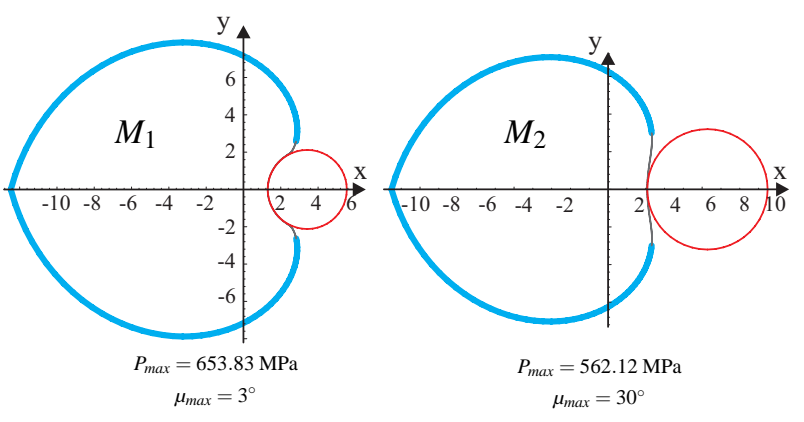

Figure 17. Optimal two conjugate-cam mechanisms
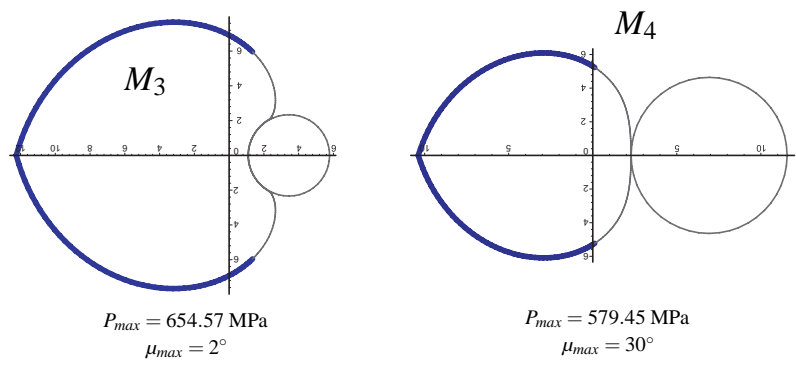

Figure 18. Optimal three conjugate-cam mechanisms

$r=4.68 \mathrm{~mm}, \mu_{\max }=2^{\circ}$ and $P_{\max }=654.57 \mathrm{MPa}$. For $M_{4}, d_{c s}=$ $4.56 \mathrm{~mm}, r=9.28 \mathrm{~mm}, \mu_{\max }=30^{\circ}$ and $P_{\max }=579.45 \mathrm{MPa}$.

According to Figs. 15 and 16, we can notice that the higher $r$, the smaller $P_{\text {max }}$. Indeed, the maximum Hertz pressure values corresponding to $M_{2}$ and $M_{4}$ are smaller than the ones corresponding to $M_{1}$ and $M_{3}$. However, the size of the mechanism along the $x$-axis is higher for $M_{2}$ and $M_{4}$. Moreover, this induces a better transmission of the torque as $d_{c s}$ is higher. Finally, we can notice that the profiles of $M_{2}$ and $M_{4}$ are easier to machine as they are fully convex.

\section{CONCLUSIONS}

The multiobjective optimization of Slide-o-Cam was reported in this paper. Slide-o-Cam is a cam mechanism with multiple rollers mounted on a common translating follower. This transmission provides pure-rolling motion, thereby reducing the friction of rack-and-pinions and linear drives. A Pareto frontier was obtained by means of a multiobjective optimization. This optimization is based on three objective functions: (i) the pressure angle, which is a suitable performance index for the transmission because it determines the amount of force transmitted to the load vs. that transmitted to the machine frame; (ii) the Hertz pressure used to evaluate the stresses produced in the contact surface between the cams and the rollers; and (iii) the size of the mechanism characterized by the number of cams and their width. It turns out that three-conjugate cam mechanisms have globally better performance that their two-conjugate cam counterparts. However, the difference is small.

\section{REFERENCES}

González-Palacios, M.A. and Angeles, J., "The design of a novel pure-rolling transmission to convert rotational into translational motion", ASME Journal of Mechanical Design, 2003, Vol. 125, pp. 205-207

Waldron, K. J. and Kinzel, G. L., Kinematics, Dynamics, and Design of Machinery, John Wiley \& Sons, Inc., New York, 1999.

Angeles, J. and López-Cajún,C., Optimization of Cam Mechanisms, Kluwer Academic Publishers B.V., Dordrecht, 1991.

Carra, S., Garziera, R. and Pellegrini, M., "Synthesis of cams with negative radius follower and evaluation of the pressure angles," Mechanism and Machine Theory, 2004, Vol. 34, pp. 1017-1032.

González-Palacios, M. A. and Angeles, J., Cam Synthesis, Kluwer Academic Publishers B.V., Dordrecht, 1993.

Lee, M.K., Design for Manufacturability of Speed-Reduction Cam Mechanisms, M.Eng. Thesis, Dept. of Mechanical Engineering, McGill University, Montreal, 2001.

Golovin A., Borisov A., Drozdova I., and Shuman B., "The simulating model of a gearing wear", Moscow State Technical University named after M.E. Bauman, Moscow, Russia, 2005.

Chablat, D. and Wenger P. (2003). Architecture Optimization of a 3-DOF Parallel Mechanism for Machining Applications, the Orthoglide. IEEE Transactions on Robotics and Automation Vol. 19/3, 403-410, June.

Chablat D. and Angeles J. "Design Strategies of Slide-o-Cam Transmission”, Proceedings of CK2005, International Workshop on Computational Kinematics, Cassino, 2005, May 4-6.

Chablat, D. and Angeles J., "The Design of a Novel Prismatic Drive for a Three-DOF Parallel-Kinematics Machine", ASME Journal of Mechanical Design, 2006, Volume 128, Issue 4, pp. 710-718, July.

Chablat, D., Caro, S. and Bouyer E. (2007) "The Optimization of a Novel Prismatic Drive taking into account the Transmitted Force", IFToMM, Besanon, France, June.

http://www.matweb.com/

Collette, Y. and Siarry, P. (2006) Multiobjective Optimization: Principles and Case Studies. Springer-Verlag Berlin Heidelberg New York. 